\title{
IMPROVING THE DURABILITY OF THE DIES AND PRODUCT QUALITY IN THE PRODUCTION OF NICHROME STRIPS
}

\author{
A. I. Snigirev, ${ }^{1}$ L. M. Zheleznyak, ${ }^{2}$ \\ N. A. Snigirev, ${ }^{3}$ and O. S. Gurtovenko ${ }^{2}$
}

UDC 669.245:621.771.23

Several complicated technological problems had to be solved to develop an alternative technology for the production of nichrome strips, the new technology involving the rolling of cast ingots and drawing of the hot-rolled semifinished products. In the traditional technology, sheet is hot-and cold-rolled and then cut lengthwise to form the strips. One particularly important advance over the existing technology is the improvement made to the geometry of the drawing channel in the hard-alloy dies. This modification significantly reduces die wear, practically eliminates breakage of the strip during drawing, and substantially improves the quality of the strip's surface.

Keywords: nichrome strips, traditional and alternative technologies, drawing, hard-alloy dies, channel geometry, tool life, reduction in breakage, improved strip quality.

Serious technological problems are encountered in the production of nichrome semifinished products of non-circular cross section (strips). In particular, nonuniform flow of metal in the width direction during flattening of the circular semifinished product in the rolls of the rolling mill causes the strip to assume a barrel shape [1]. The tool does not control the convexity of the small sides of the strip, so that it can vary in relation to the a number of process variables: the wear of the rolls; the size of the reduction being made; the character of the friction, etc. Thus, it is technically difficult to roll strips within the tolerance and keep the deviations in cross-sectional area along the strip to a minimum. Keeping the cross-sectional area constant is important for the reliable operation of nichrome heaters, since the metal overheats, is oxidized, becomes saturated with gases, and eventually loses the necessary service properties when current passes through parts of the heater with a smaller cross section, Thus, to ensure the long-term operation of such heaters, the strips which serve as the semifinished products must have stable physico-mechanical characteristics over their length. Such stability is assured by having the transverse dimensions of the strip be constant. This prerequisite is particularly important from the standpoint of maintaining a stable temperature regime in metallurgical resistance furnaces with nichrome heaters.

In the technology that has traditionally been used, the nichrome semifinished products are made from sheet or wide strip [2-5] produced by flat rolling and are then cut into strips of the required width. After being cut lengthwise, the cross section of the finished strip has the form of a rectangle without a radius of curvature at the corners; in addition, the Russian standard [6] allows the formation of projections on the edges of the strip. The projections cannot be any larger than the sum of the maximum permissible thickness deviations. This sum ranges from $\pm 0.045 \mathrm{~mm}$ to $\pm 0.080 \mathrm{~mm}$ for strips with a thick-

\footnotetext{
${ }^{1}$ Zavod Uralprokat, Kamensk-Uralsky, Russia.

${ }^{2}$ Yeltsin Ural Federal University (UrFU), Ekaterinburg, Russia; e-mail: bsp@mtf.ustu.ru.

${ }^{3}$ Kamensk-Uralsky Nonferrous Metals Plant, Kamensk-Uralsky, Russia.
}

Translated from Metallurg, No. 8, pp. 92-96, August, 2013. Original article submitted December 27, 2012. 


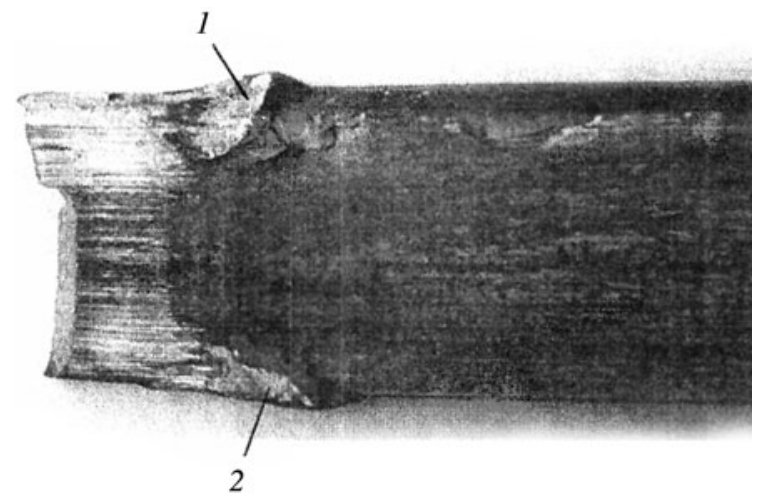

Fig. 1. Semifinished product that could not be drawn due to its fracture: 1,2) "excess" volumes of metal in the form of waves (ridges).

ness in the range $h=1.0-3.2 \mathrm{~mm}$. The presence of such sharp edges - especially edges with projections - poses a danger to personnel engaged in the operations of bending the metal and fabricating and installing the heating element.

Since the volume of orders that ferrous metallurgy plants receive from Russian customers for nichrome strips has declined substantially over the last 20 years, it has become unprofitable to make these products by the traditional technology - which entails the flat rolling of ingots with a weight on the order of $500 \mathrm{~kg}$. An alternative technology has thus been developed to make commercial batches of strips composed of nichrome of grade Kh20N80-N. The technology includes the hot rolling of ingots (cast in ingot molds) into light (55-60 kg) sections and multi-pass drawing of the hot-rolled semifinished products with intermediate annealings to obtain strips of the prescribed dimensions [7,8]. The modified hard-alloy drawing die that has proven to be expedient for making high-quality electrical-grade strips of copper and copper alloys is not suited for drawing strips made of nichrome. Thus, recommendations were developed to improve the parameters of the drawing channel in order to allow the tool to be used to draw nichrome strips. The quality of the resulting product turns out to exceed the requirements of the standard, since the new technology also ensures workers' safety by eliminating projections from the strips' edges.

In accordance with the patent [9], in order to obtain rectangular strips with a radius of curvature of $0.5-2.0 \mathrm{~mm}$ at the corners, the channel in the die should have the same radii at the corners in the working zone as well as the sizing zone. Otherwise, the laminar flow of the metal would be disturbed during deformation. Such a development would be highly undesirable for the drawing of nichrome, which has a high resistance to deformation in the strain-hardened state (up to 1200-1300 MPa) and has a strong tendency to adhere to the surface of the die's hard alloy.

Figure 1 shows the non-elongated back end of a semifinished product extracted from the channel after fracture of the strip. "Excess" volumes of metal can be seen on the end in the form of waves (ridges) 1 and 2 . These waves extend beyond the large and small surfaces of the strip because there is no space for the metal to flow into at the corners of the working zone when the radius $r \approx 1 \mathrm{~mm}$, i.e., when the radius is within the range recommended in [9]. This is consistent with several problems that have been seen in the factory production of nichrome strips: rapid wear of the channel in the working and sizing zones from a lack of lubricant at the locations where ridges are formed due to the excessive radius of curvature of the channel's corners; frequent fracture of the strips; a deterioration in the quality of the strips' surface.

Since there is no standard that governs the production of cold-drawn nichrome strips, the normative document is the above-mentioned standard for cold-rolled sheet [6]. In accordance with the latter, although the entire thickness range specified for the sheet is $0.1-3.2 \mathrm{~mm}$, realistically only strips having a thickness within the range $1.0-3.2 \mathrm{~mm}$ can be obtained by drawing. This stems from the fact that nichrome strips with a thickness $h<1.0 \mathrm{~mm}$ are technically extremely difficult to draw because of the features of deformation of nichrome. Calculations show that for $r_{\min }=0.5 \mathrm{~mm}$ the ratio $\mathrm{r}_{\min } / h=0.5 /(1.0-3.2)=$ $=0.5-0.156$. Values in the latter range should be considered excessively high from the standpoint of disturbing the pattern of flow of the metal (see Fig. 1). 

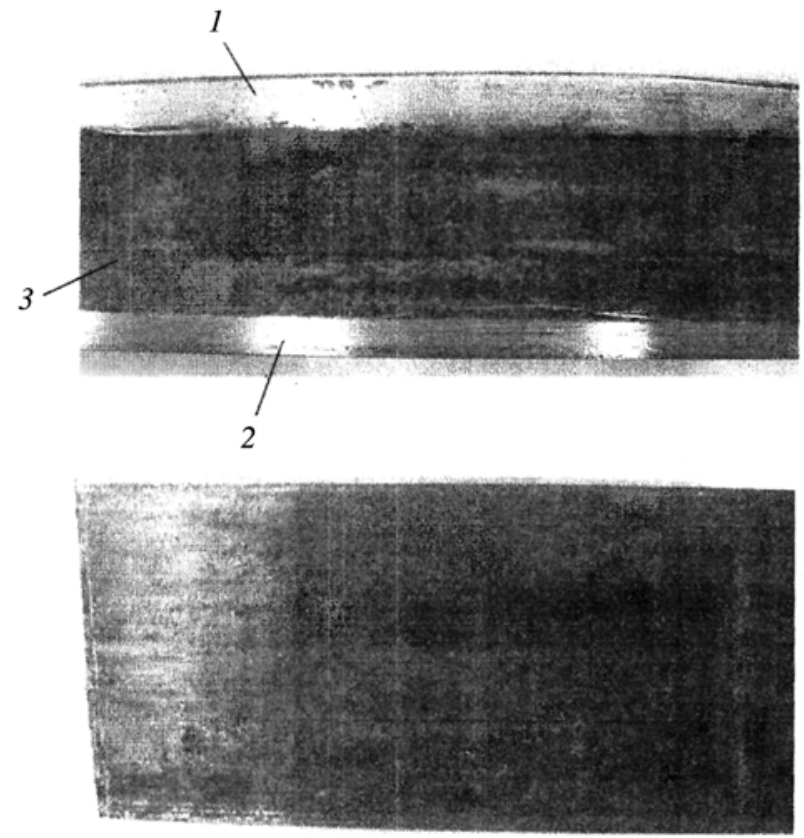

$b$

Fig. 2. Condition of the surface of nichrome strips: $a$ ) with shiny lengthwise sections 1 and 2 and dull surface 3 on the large surface of the strip; $b$ ) completely dull large surface.

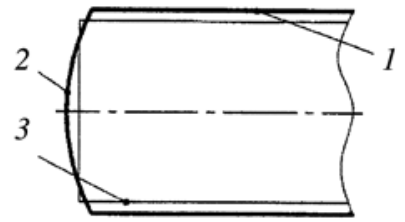

$a$

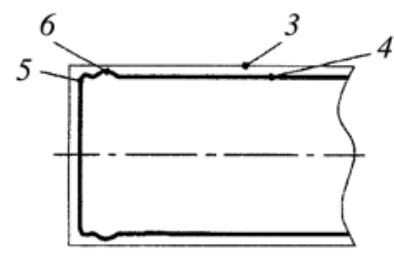

$b$

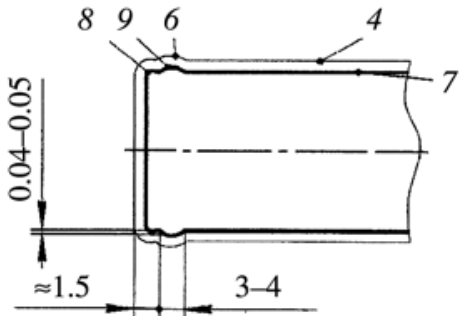

$c$

Fig. 3. Schematic representation of fragments of the contours of the cross section of semifinished products and a finished strip, $\times 10: a$ ) situation in the first drawing pass; $b$ ) second, leader pass; $c$ ) third, finishing pass; 1) contour of the cross section of a hot-rolled semifinished product with lateral convexity 2 resulting from free spreading during rolling; 3 ) contour of the cross section of the semifinished product after the first pass; 4) contour of the cross section of the semifinished product in the leader pass with rounded corners 5 and projection of the metal 6 on the side of the strip; 7) contour of the cross section of the finished strip after three passes, with rounded corners 8 and ridge 9 .

To improve the surface quality of nichrome strips, reduce the incidence of fracture, increase productivity, and reduce wear of the hard-alloy dies, the following recommendation has been developed and validated in practice [10]: the radius of curvature at the corners of the working zone of the drawing channel should be kept within a range of values equal to $0.125-0.039$ of the thickness of the cross section of the sizing zone, i.e., the condition $r \leq(0.125-0.039) h$ must be observed.

The presence of lustrous lengthwise sections 1 and 2 on the large surface of the drawn strip (Fig. $2 a$ ) indicates that there was not any lubricant on the strip's surfaces as it was drawn through the die when $r / h \approx 0.25$. This situation is in contrast to the dull surface 3 , which shows that there was enough lubricant to create a boundary friction regime. Figure $2 b$ shows 


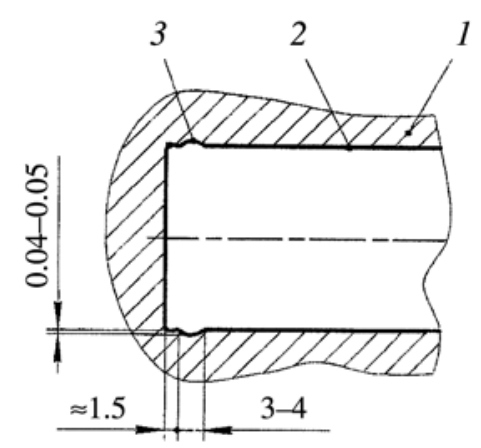

Fig. 4. Schematic representation of a fragment of the cross section of a hard-alloy die 1 in the sizing zone 2 with a characteristic type of wear in the form depressions 3 in the alloy when $r / h \approx 0.3$ in accordance with [9].

the completely dull large surface of the strip drawn with $r / h \approx 0.042$, i.e., within the range prescribed in [10]. With 10-fold magnification, Fig. 3 schematically depicts fragments of the contours of the cross section of semifinished products and a finished strip that reflect the character of metal flow after three drawing passes made with $r / h \approx 0.25$ in accordance with [9].

The above-described deformation conditions facilitated high-rate local wear of the channel's sizing zone in the form of depressions 3 of the corresponding dimensions (Fig. 4). The die was removed from service when ridges having a combined thickness $\geq 0.08 \mathrm{~mm}$ were formed on the large surfaces of the finished strip, since - as noted above - the thickness tolerance is $\pm 0.08 \mathrm{~mm}$ [6]. Observing this tolerance is especially important for $h=2.5-3.2 \mathrm{~mm}$ (i.e., for the commercial batches made in the largest quantities). Ridges are usually formed in the second and third passes (in the case of a 4-5-pass drawing schedule), since the amount of space available to accommodate the volumes of metal that are being deformed is not sufficient to eliminate the barrel shape of the hot-rolled semifinished product and ensure that its cross section is close to rectangular when the ratio $r / h$ is too large [9].

The desired profile of the channel can be obtained with a high degree of accuracy by resorting to electro-erosive cutting on high-precision machine tool AGIECUT CLASSIC 2S (Switzerland) and using 0.25-mm-diam. wire of brass L63 as the consumable electrode. A radius of curvature equal to $0.25 / 2=0.125 \mathrm{~mm}$ is obtained automatically at the corners of the working and sizing zones during the machining of the channel. For nichrome strips with a thickness in the range 1.0-3.2 mm [6], the upper and lower limits of the ratio $r / h$ are respectively $(r / h)_{\max }=0.125 / 1.0=0.125$ and $(r / h)_{\min }=0.125 / 3.2=0.039$, i.e., they conform to the recommendations in [10].

The formation of a channel with the parameters specified in [10] led to a substantial reduction in its wear by eliminating the formation of ridges on the large and small surfaces of the strip. No ridges were formed because the corners of the cross section of the working and sizing zones were filled with metal. The modification also prevented the formation of the shiny lengthwise sections that are seen on the strips when there is no lubricant, i.e., the change created a stable boundary-friction regime. Thus, solutions were found to the problems of reducing tool wear, eliminating fracture of the strips, and improving the quality of the strips' surface. The dies now have a maximum service life of 3.5-4.0 tons of drawn metal, which is in sharp contrast to the situation that existed with the previous tool (when tool life was $500 \mathrm{~kg}$ of metal in the best-case scenario). Previously, the drawing operation was often interrupted by fracture of the front end of the strip and all of the associated consequences. The most negative effect of these developments was a drop in productivity.

As regards the case in which the front of the strip fractures because its safety factor $k=\sigma_{\mathrm{ul}} / p$ is exceeded (where $\sigma_{\mathrm{u} 1}$ is the tensile strength of the strain-hardened alloy as a function of the degree of deformation; $p$ is the drawing stress), the condition $k \geq 2.0-2.5$ should be observed because there are always negative factors at work in the actual production process: deviations of the annealing regime from the norm; incomplete removal of scale in the pickling operation; an inadequate supply of lubricant or the use of an inappropriate lubricant (powdered sodium soap with additives); disturbance of the coaxiality of the die channel and the direction of the drawing force; vibration of the pulling mechanism of the drawing machine 
or other dynamic effects, etc. The use of a tool having a channel that is incorrect based on the data in [10] results in the formation of ridges which significantly increase the likelihood of fracture. This has been proven to be the case in the factory production of nichrome strips.

We should mention one possible adverse consequence of decreasing the ratio $r / h$ : the corners of a channel with a reduced radius of curvature become a stress concentrator, and under unfavorable conditions that can cause cracks to form at the corners. It has been found that cracks occasionally form in the corners after the drawing of 200-300 $\mathrm{kg}$ of metal (which corresponds to 4-6 50-kg coils) and that this usually takes place during the second and third passes. Ridges that have a thickness of about $0.05 \mathrm{~mm}$ and a length of $1.0-1.5 \mathrm{~mm}$ and are formed on the edges of the semifinished product due to the flow of metal into the cracks are eliminated by intermediate annealings and subsequent picklings. It has also been observed that the cracks "close up" after the drawing of a certain amount of metal. The exact amount of metal in question is difficult to determine. The closure of the cracks in this case is probably attributable to the pronounced adhesiveness of the chromiumnickel alloy, which fills the crack.

Conclusion. Fourteen grades of nichrome Kh20N80-N strips that fully meet the requirements of the standard [6] are now being successfully made on an industrial scale. It is particularly noteworthy that some of these strips are being produced in the soft, heat-treated state and have a resistivity $\rho=1.06-1.17 \mu \Omega \cdot \mathrm{m}$. By controlling the range of values for the ratio of the radii of curvature of the corners of the working and sizing zones of the drawing channel to the thickness of the strip, it has become possible to make the channel more resistant to wear and improve the surface quality of the finished strip. This has also eliminated fractures of the strips and the danger formerly posed to personnel during bending of the strips and the installation of heating elements in resistance furnaces.

\section{REFERENCES}

1. U.S. Patent No. 4552599, IPC B21C37/04, H01B13/00, Process for Producing Insulated Rectangular Wire, publ. 11.12.1985.

2. U. S. Patent No. 3177075, IPC 75-171, Nickel-Chromium Sheet Alloy, publ. 04.06.1965.

3. U. S. Patent No. 3625678, IPC C22C19/00, Nickel-Chromium Sheet Alloy Adapted for Producing Weldable Sheet, publ. 12.07.1971.

4. French Patent No. 2745147, IPC H05B3/30, Resistive Heating Element Enclosed in a Layered Mica Sheet, publ. 08.22.1997.

5. $\quad$ British Patent No. 2153845, IPC C22C19/05, Production of Superalloy Sheet, publ. 08.29.1985.

6. GOST 12766.2-90, Sheet Made of Precision Alloys with a High Resistivity.

7. N. S. Arsent'eva, L. M. Zheleznyak, N. F. Bokov, et al., Russian Patent No. 2385778, IPC B21B3/00, Method of Making Strips of Nichrome and Similar Alloys, subm. 01.29.2008, publ. 04.10.2010, Byull., No. 10.

8. N. A. Arsent'eva, A. I. Snigirev, L. M. Zheleznyak, et al., "Production of different types of wire and strip at the Kamensk-Uralsky Nonferrous Metals Plant,” Metallurg, No. 7, 70-74 (2006).

9. A. I. Snigirev, P. A. Vasilevskii, and Yu. N. Loginov, Russian Useful-Model Patent No. 32495, IPC ${ }^{7}$ C22C19/05, Strips of Nichrome and Similar Alloys, subm. 03.11.2003, publ. 09.20.2003, Byull., No. 26.

10. N. S. Arsent'ev, L. M. Zhleznyak, A.I. Snigirev, and N. A. Snigirev, Russian Patent No. 2371267, IPC B21C3/00, Hard-Alloy Die for Drawing Nichrome Strips, subm. 02.04.2008, publ. 10.27.2009, Byull., No. 30. 